\title{
1,3-propanediol production by Escherichia coli expressing genes of dha operon from Clostridium butyricum 2CR371.5
}

\author{
Sławomir Dąbrowski ${ }^{凶}$, Dorota Pietrewicz-Kubicz, Ewa Zabłotna and Anna Długołęcka \\ A\&A Biotechnology, Gdynia, Poland
}

1,3-propanediol is used as a monomer in the production of some polymers e.g. polytrimethylene terephthalate used in the production of carpets and textile fibers and in the thermoplastics engineering. However, the traditional chemical synthesis is expensive, generates some toxic intermediates and requires a reduction step under high hydrogen pressure. Biological production of 1,3-propanediol could be an attractive alternative to the traditional chemical methods. Moreover, crude glycerol which is a by-product of biodiesel production, can be used. We constructed a recombinant Escherichia coli strain producing 1,3-propanediol from glycerol by introducing genes of the dha operon from Clostridium butyricum 2CR371.5, a strain from our collection of environmental samples and strains. The $E$. coli strain produced $3.7 \mathrm{~g}$ of 1,3-propanediol per one litre of culture with the yield of $0.3 \mathrm{~g}$ per $1 \mathrm{~g}$ of glycerol consumed.

Key words: 1,3-propanediol, anaerobic fermentation, biodiesel, Clostridium butyricum, dha operon, Escherichia coli, glycerol

Received: 12 February, 2012; revised: 16 July, 2012; accepted: 30 July, 2012; availabler on-line: 01 August, 2012

\section{INTRODUCTION}

1,3-propanediol (1,3-PDO) is used as a monomer in the production of several types of polymers, one of which is polytrimethylene terephthalate (PTT), produced by polycondensation of 1,3-PDO with terephtalic acid or its esters. PTT is a type of polyester used in the production of carpets and textile fibers and in thermoplastics engineering (Liu et al., 2010).

1,3-PDO can be produced in many ways i.a. from ethylene oxide over a catalyst in the presence of phosphine, water, carbon monoxide, hydrogen and an acid. However, the traditional chemical synthesis is expensive, generates some toxic intermediates and requires a reduction step under high hydrogen pressure (Raynaud et al., 2003; Emptage et al., 2009). Biological production of 1,3-PDO could be a useful alternative to the traditional chemical methods because of its numerous advantages, including a lower environmental impact. Moreover, crude glycerol, which is a by-product of biodiesel production, can be used as precursor. The conversion of glycerol to highervalue products, such as 1,3-PDO should decrease the cost of biofuel production (Zeng \& Biebl, 2002; Yazdani \& Gonzalez, 2007).

Several bacterial species fermenting glycerol to 1,3PDO are known e.g., Lactobacillus sp., Citrobacter freundii, Clostridium sp., Klebsiella sp. (Boenigk et al., 1993; Luo et al., 2011; Mu et al., 2006; Burkhard et al., 2009; Pasteris et al., 2009; Gungormusler et al., 2010; Chatzifragkovet et al., 2011; Zheng et al., 2008). Additionally, genetically modified E. coli, Saccharomyces cerevisiae and Pichia pastoris strains can also be used (Burch et al., 1997; Nagarajan \& Nakamura, 1997; Nevoigt, 2008). However, traditional methods of 1,3-PDO production are still much more effective than the biological one.

Glycerol fermentation by glycerol-fermenting microorganisms is a two-branched pathway. The 1,3-PDO production by $C$. butyricum is the reductive branch catalysed by two enzymes, (i) glycerol dehydratase encoded by dhaB1 and (ii) NADH-dependent 1,3-PDO oxidoreductase (encoded by dhaT), with 3-hydroxypropionaldehyde as an intermediate (Fig. 1). Glycerol dehydratase requires for activity the presence of a protein encoded by the dhaB2 gene. All three genes are located in the dha operon, the expression of which is induced in the presence of dihydroxyacetone or glycerol. On the other hand, in the oxidative branch, glycerol is dehydrogenated by NAD-dependent glycerol dehydrogenase to dihydroxyacetone (DHA). DHA is then converted sequentially to glyceraldehyde-3-phosphate, phosphoenolpyruvate, and pyruvate (Saint-Amans et al., 2001; Raynaud et al., 2003; Daniel et al., 2005; Gupta et al., 2009; Marçal et al., 2009).

Due to the lack of the $d h a$ operon E. coli cannot ferment glycerol to 1,3-PDO in the absence of an external electron acceptor. Instead 1,2-PDO and ethanol are produced (Tong et al., 1991; Gupta et al., 2009). However, genetically modified E. coli strains fermenting glycerol to 1,3-PDO have been constructed (Daniel et al., 1995; Emptage et al., 2009; Eliot et al., 2011).

Here we report construction of a recombinant E. coli strain producing 1,3-PDO from glycerol by introducing $d h a B 1, d h a B 2$, and $d h a T$ genes from the $d h a$ operon from C. butyricum 2CR371.5, a strain from our collection of environmental samples and strain. The strain was previously isolated and tested as a 1,3-PDO producer and the results were described in the accompanying article (Dabrowski et. al, 2012).

\section{MATERIALS AND METHODS}

Bacterial strains and growth conditions. C. butyricum 2CR371.5 from our collection was used as the source of $d h a$ operon containing $d h a B$ and $d h a T$ genes. It was cultured anaerobically at $37^{\circ} \mathrm{C}$ for 3 days in a medium containing: Yeast Nitrogen Base without amino acids and without ammonium sulphate (Formedium) - $6.9 \mathrm{~g} \cdot \mathrm{l}^{-1}, \mathrm{NaHCO}_{3}-2.6 \mathrm{~g} \cdot \mathrm{l}^{-1}$, yeast extract -2

e-mail: sd@aabiot.com

Accession number (GenBank): JQ346526

Abbreviations: 1,3-PDO, 1,3-propanediol; 3-HPA, 3-hydroxypropionaldehyde; DSMZ, Leibnitz-Institut Deutsche Sammlung von Mikroorganizmen und Zellkulturen $\mathrm{GmbH}$ 
$\mathrm{g} \cdot \mathrm{l}^{-1}$, cysteine $-0.5 \mathrm{~g} \cdot \mathrm{l}^{-1}$, resazurin $-0.5 \mathrm{mg} \cdot \mathrm{l}^{-1}$, $\mathrm{MgCl}_{2} \cdot 6 \mathrm{H}_{2} \mathrm{O}-0.9 \mathrm{~g} \cdot \mathrm{l}^{-1}$, glycerol $-10 \mathrm{~g} \cdot \mathrm{l}^{-1}, \mathrm{ZnCl}_{2}$ - $50 \mathrm{ng} \cdot \mathrm{l}^{-1}, \mathrm{MnCl}_{2} \cdot \mathrm{H}_{2} \mathrm{O}-38 \mathrm{~g} \cdot \mathrm{l}^{-1}, \mathrm{CoCl}_{2} \cdot 2 \mathrm{H}_{2} \mathrm{O}$ - $50 \mathrm{ng} \cdot l^{-1}, \mathrm{NiCl}_{2} \cdot 6 \mathrm{H}_{2} \mathrm{O}-92 \mathrm{ng} \cdot \mathrm{l}^{-1}, \mathrm{FeCl}_{2} \cdot 2 \mathrm{H}_{2} \mathrm{O}-$ $2.3 \mathrm{mg} \cdot \mathrm{l}^{-1},(\mathrm{NH})_{4} \mathrm{Mo}_{7} \mathrm{O}_{24} \cdot 4 \mathrm{H}_{2} \mathrm{O}-50 \mathrm{ng} \cdot \mathrm{l}^{-1}$, EDTA - $0.5 \mathrm{mg} \cdot \mathrm{l}^{-1}$. An anaerobic atmosphere was generated by using Biogon ${ }^{\circledR} \mathrm{C}-20\left(80 \% \mathrm{~N}_{2}, 20 \% \mathrm{CO}_{2}\right)$. All experiments in anaerobic conditions were performed in a MiniMACS anaerobic workstation (Don Whitley Scientific).

E. coli TOP10F' (Invitrogen) was used as the host for cloning and the resulting transformants were grown at $37^{\circ} \mathrm{C}$ for $16-18 \mathrm{~h}$ in Luria-Bertani (LB) broth containing ampicillin $\left(50 \mu \mathrm{g} \cdot \mathrm{ml}^{-1}\right)$.

Strains E. coli BL21(DE3) and Rosetta2(DE3) (Novagen) were used as hosts for expression of the dhaB1, dhaB2 and dhaT genes. They were grown at $37^{\circ} \mathrm{C}$ in $\mathrm{LB}$ medium containing glycerol $\left(10 \mathrm{~g} \cdot \mathrm{l}^{-1}\right)$ and eventually also glucose $\left(10 \mathrm{~g} \cdot \mathrm{l}^{-1}\right)$. The solid medium and liquid medium for non-induced overnight culture did not contain glucose or glycerol. LB medium for E. coli Rosetta2(DE3) was additionally supplemented with $50 \mu \mathrm{g} \cdot \mathrm{ml}^{-1}$ of chloramphenicol and media for culturing transformants were also supplemented with $50 \mu \mathrm{g} \cdot \mathrm{ml}^{-1}$ ampicillin.

DNA manipulation. DNA manipulations were carried out according to standard procedures (Sambrook \& Russel, 2001) or manufacturer's recommendations. Restriction endonucleases were purchased from Fermentas, T4 DNA ligase was from A\&A Biotechnology. To purify DNA after enzymatic reactions DNA Clean-up AX and DNA Gel-out kits (A\&A Biotechnology) were used. To isolate plasmid and genomic DNA Plasmid Mini AX and Genomic Mini AX Bacteria Spin (A\&A Biotechnology), respectively, were used.

Sequencing and ORF analysis. Genomic DNA of C. butyricum 2CR371.5 was partially sequenced (Macrogen). The obtained genomic sequence fragments were analysed using the CLC Genomics Workbench software. To identify the DNA fragment encoding 1,3-propanediol operon components, the obtained C. butyricum 2CR371.5 sequence was compared with the nucleotide sequence of the 1,3-propanediol operon of C. butyricum DSM 2478 (GenBank: DQ901407.2).

Sequence similarity analysis was done with the BLASTN and BLASTX programs using the server at the National Center of Biotechnology Information (http:// blast.ncbi.nlm.nih.gov/Blast.cgi).

Construction of expression plasmid. Plasmid pUC57 (Fermentas) was used for construction of the expression vector.

For amplification of the $\mathrm{d} h a B 1$ and $d h a B 2$ genes as a single fragment from C. butyricum 2CR371.5, DNA primers ForDhaB1SphI 5'-GCG GCG GCA TGC GTG AT'T GAA GGA GTA AAA ATG ATA AG-3' and RevDhaB2SalI 5'-GCG GCG GTC GAC GTA AAG CTA CTA T'TA CTC AGC TCC-3' were used. The obtained PCR product of 3360 bp was cloned into SphI and SalI sites of pUC57 vector which resulted in pUCdhaB1B2 plasmid.

To clone the dhaT gene, primers ForDhaB2BamHI 5'GCG GCG GGA TCC AAG GAG ATA AAA GTA ATG AGT AAG G-3' and RevDhaTKpnI 5'-GCG GCG GGT ACC T'T'T TAC TT'T GAA TCC T'T'T AAA TAG-3' were used. The PCR product of $2201 \mathrm{bp}$ was then digested with $A f l \mathrm{II}$ and $K \not n \mathrm{I}$ and cloned into pUCdhaB1B2. The resulting plasmid was named pUCdhaB1B2T (Fig. 2). Sequence correctness of the constructed plasmid was confirmed by sequencing (Macrogen) with the use of universal primers M13F-pUC and
M13R-pUC (Macrogen). Sequences of specific recognition sites in the primers used are underlined.

The PCR reaction profile was: (i) initial denaturation $94^{\circ} \mathrm{C}$ for $2 \mathrm{~min}$; (ii) 30 repeats: $94^{\circ} \mathrm{C}$ for $30 \mathrm{~s}, 65^{\circ} \mathrm{C}$ for $30 \mathrm{~s}, 72^{\circ} \mathrm{C}$ for $2 \mathrm{~min}$; (iii) final extension $72^{\circ} \mathrm{C}$ for $5 \mathrm{~min}$. The reaction was performed with the use of $2 \times \mathrm{PCR}$ Master Mix Plus High GC (A\&A Biotechnology).

Expression in $\boldsymbol{E}$. coli. E. coli BL21(DE3) or Rosetta2(DE3) cells were transformed with pUCdhaB1B2T.

Cells were grown aerobically for $16 \mathrm{~h}$ at $37^{\circ} \mathrm{C}$ in $\mathrm{LB}$ medium containing antibiotics. The preculture was then inoculated $(1 \%)$ into $100 \mathrm{ml}$ of fresh LB medium with antibiotics, glycerol or glycerol plus glucose. The cultivation was at $37^{\circ} \mathrm{C}$ until optical density at $600 \mathrm{~nm}$ of 0.5 was reached. The culture was then supplemented with $0.5 \mathrm{mM}$ isopropyl- $\beta$-D-thiogalactopyranoside (IPTG) and grown without agitation for 2 weeks. To limit the oxygen concentration, the induced cultures were grown in anaerobic flasks.

As references, non-induced cultures and $E$. coli BL21(DE3) and Rosetta2(DE3) transformed with pUC57 were used.

To test the influence of iron ion on the 1,3-PDO production the liquid medium was supplemented with $\mathrm{FeCl}_{2}$ up to $100 \mu \mathrm{M}$.

Analytical methods. HPLC analyses were performed using a HPLC Agilent 1200 Series system with RID and DAD detectors, a Phenomenex Rezex ROA column $\left(300 \times 7.80 \mathrm{~mm} ; 8\right.$ microns) with a $3 \mathrm{mM} \mathrm{H}_{2} \mathrm{SO}_{4}$ as the eluent $\left(0,6 \mathrm{ml} \cdot \mathrm{min}^{-1}\right)$ and the column temperature of $60^{\circ} \mathrm{C}$.

For quantitative determination of 3-HPA a colorimetric method described by Krauter et al. (2012) was used. The absorbance was measured at $650 \mathrm{~nm}$ (Perkin Elmer Lambda EZ 150).

\section{RESULTS}

\section{Analysis of the dha operon}

A genomic DNA fragment containing the 1,3-propanediol operon (dha operon) of $C$. butyricum 2CR371.5 was identified using the CLC Genomic Workbench. It was submitted to GenBank with the accession number JQ346526. The nucleotide sequence of this fragment shows the identity with $99 \%$ to the corresponding operon of C. butyricum DSM 2478. We found three distinct open reading frames encoding DhaB1, DhaB2 and DhaT, components of the dha operon of $C$. butyricum 2CR371.5. DhaB1, comprising of 2,361 nucleotides, encodes a protein of 787 amino acids. The deduced amino acid sequence shows the highest identity $(100 \%)$ with the amino acid sequence of $\mathrm{B}_{12}$-independent glycerol dehydratase from C. butyricum. DhaB2, of 912 nucleotides, encodes a protein of 304 amino acids. The deduced amino acid sequence shows $100 \%$ identity with the amino acid sequence of glycerol dehydratase activator from C. butyricum. DhaT, of 1,155 nucleotides, encodes a protein of 385 amino acids. The deduced amino acid sequence shows $100 \%$ identity with the amino acid sequence of 1,3-propanediol dehydrogenase from C. butyricum.

\section{Production of 1,3-PDO by $E$. coli}

The growth of all the E. coli strains harbouring the pUCdhaB1B2BT plasmid was much slower comparing 


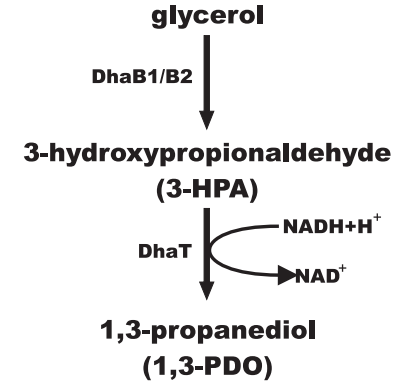

Figure 1. Glycerol fermentation pathway in anaerobic bacteria. DhaB1, glycerol dehydratase; DhaB2, glycerol dehydratase activating protein; DhaT, 1,3-propanediol oxidoreductase.

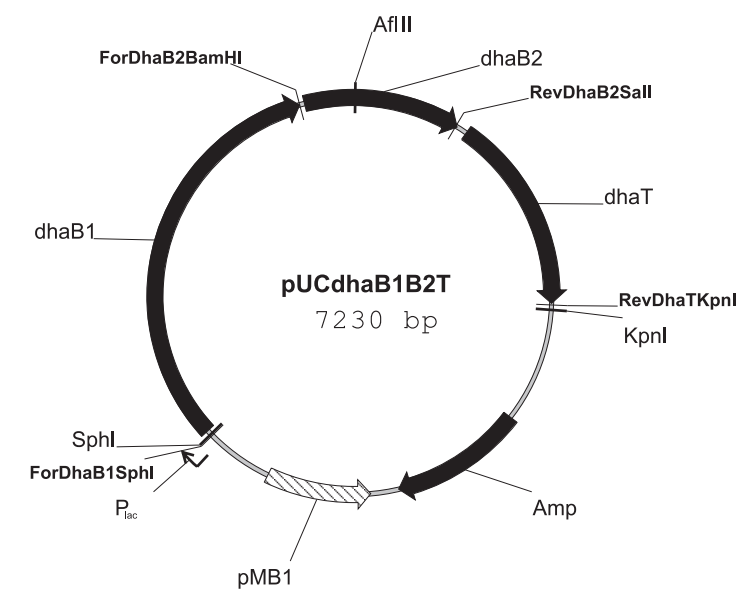

Figure 2. A map of the pUCdhaB1B2T plasmid.

Amp, ampicilin resistance gene; $P_{\text {lac }}$ lactose promoter; $\mathrm{pMB1}$, replicon; Afll, Kphl, Sphl, restriction endonuclease sites.

with control E. coli strains transformed with pUC57 plasmid.

Seven days after expression induction with IPTG the E. coli strains BL21(DE3) and Rosetta2(DE3) transformed with pUCdhaB1B2T produced 1,3-PDO in the concentration of $0.1 \mathrm{~g} \cdot \mathrm{l}^{-1}$ (not shown).

The HPLC analysis performed two weeks after IPTG induction showed 1,3-PDO concentration of $0.34 \mathrm{~g} \cdot \mathrm{l}^{-1}$ in the case of E. coli BL21(DE3) and $3.7 \mathrm{~g} \cdot \mathrm{l}^{-1}$ in the case of E. coli Rosetta2(DE3) when glycerol was used as the sole carbon source (Fig. 3).

When a medium containing both glycerol and glucose was used, the concentration of 1,3-PDO two weeks after IPTG induction did not exceed $0.3 \mathrm{~g} \cdot \mathrm{l}^{-1}$ for either of the two E. coli strains (Fig. 3).

The yield of the 1,3-PDO production relative to the amount of glycerol consumed was up to $0.028 \mathrm{~g}$ per one gram of glycerol for eE. coli BL21(DE3) and up to $0.3 \mathrm{~g}$ for Rosetta2(DE3) (Fig. 4).

Table 1. 3-hydroxypropionaldehyde and 1,3-propanediol production by $E$. coli Rosetta2(DE3) cells expressing genes of dha operon of C. butyricum 2CR371.5 in LB medium supplemented with glycerol.

The increase of 1,3-PDO and 3-HPA was calculated as the ratio of the product amount at a given time to that at the previous time analysed.

\begin{tabular}{lllll}
\hline Days after induction with IPTG & $\begin{array}{c}1,3-P D O \\
{\left[\mathrm{~g} \cdot \mathrm{I}^{-1}\right]}\end{array}$ & $1,3-\mathrm{PDO}$ increase & $\begin{array}{c}3-\mathrm{HPA} \\
{\left[\mathrm{g} \cdot \mathrm{I}^{-1}\right]}\end{array}$ & 3-HPA increase \\
\hline 3 & 0.21 & - & 0.12 & - \\
7 & 0.74 & 3.52 & 0.62 & 5,17 \\
10 & 2.21 & 2.99 & 2.71 & 4,37 \\
14 & 3.72 & 1.68 & 5.24 & 1,93 \\
\hline
\end{tabular}

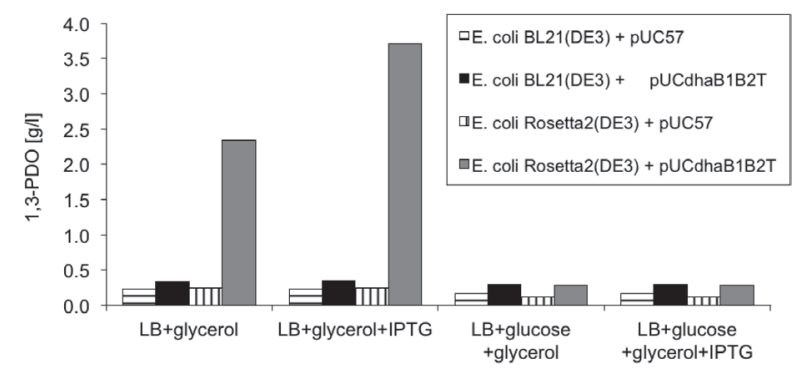

Figure 3. 1,3-propanediol concentration after two weeks of expression expressing dha genes of 2CR371.5 in E. coli.

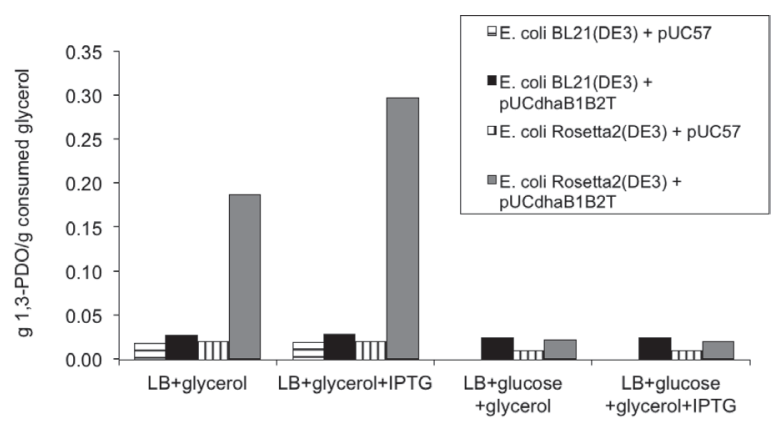

Figure 4. 1,3-propanediol production per consumed glycerol, after two weeks of expression dha genes of 2CR371.5 in E. coli.

Simultaneously with 1,3-PDO the amount of 3-HPA was also measured, following induction with IPTG its production increased more than that of 1,3-PDO (Table 1).

Iron ions at concentration up to $100 \mu \mathrm{M}$ had no effect on 1,3-PDO production (not shown).

\section{DISCUSSION}

There are several microorganisms naturally fermenting glycerol to 1,3-PDO, but their large-scale application is limited due to some difficulties. The main problems are (i) accumulation of by-products which are toxic and can inhibit 1,3-PDO production; (ii) some of these microorganisms are human pathogens; (ii) most of the 1,3-PDOproducing bacteria need $\mathrm{B}_{12}$ vitamin as a cofactor for glycerol dehydratase (DhaB) which increases costs $(\mathrm{Na}-$ kamura \& Whited, 2003; O'Brien et al., 2004). Construction of genetically modified $E$. coli strains express $\mathrm{B}_{12^{-}}$ independent glycerol dehydratase gene could overcome these difficulties.

We constructed a system for heterologous expression in E. coli genes of the glycerol of metabolic pathway from C. butyricum 2CR371.5, from the A\&A Biotechnology collection. The cloned genes dhaB1, dhaB2 and dhaT encode a vitamin $\mathrm{B}_{12}$-independent glycerol dehydratase, its activating factor and an NADHdependent 1,3-PDO oxidoreductase, respectively.

The first problem we came across was the poor growth of the E. coli strains harbouring the pUCdhaB1B2T plasmid. It can be 
explained by the fact that the LB medium could contain a small amount of lactose to induce expression of the cloned genes under the lac promoter at a low level. That in turn would in accumulation of an intermediate (3-HPA), final product (1,3-PDO), or heterologous protein/-s (DhaB1, DhaB2 or DhaT). We suppose the agent which caused the growth inhibition was 3-HPA, because it was proved previously to be toxic to bacterial cells (Hao et al., 2008b).

The observed 1,3-PDO production by E. coli Rosetta2(DE3) was about 11-fold higher comparing with E. coli BL21(DE3) (Fig. 3). It can be explained by the fact that E. coli Rosetta2(DE3) contains a plasmid conferring chloramphenicol resistance that supplies rare tRNAs. Consequently, translation is not limited by the codon usage of $E$. coli (Kane, 1995). Analysis of the predicted amino acid sequences of DhaB1, DhaB2 and Dha'T revealed that they contain 14, 12 and $14 \%$ of rare codons, respectively.

The low amount of 1,3-PDO produced in our expression system could be correlated with 3-HPA concentration. 3-HPA, an intermediate of 1,3-PDO production, is an inhibitor of DhaB1 (Barbirato et al., 1996a; Hao et al., 2008a; 2008b). The 3-HPA accumulation is correlated with a higher NAD/NADH ratio and it is known that NAD behaves as a competitive inhibitor of glycerol dehydratase (Barbirato et al., 1996b). The level of 3-HPA in culture medium could be controlled by the substrate (glycerol) concentration, and a lower level of glycerol could prevent 3-HPA accumulating to a high, lethal concentration (Hao et al., 2008). 3-HPA accumulates when the first step of glycerol fermentation to 1,3-PDO is faster than the second one, e.g. when the activity of DhaT is lower than that of DhaB1. Such a situation often occurs in polycistronic expression systems where the mRNA lacks internal ribosome entry sites for the next cistrons (Nishizawa et al., 2010). That was not the case in our expression system, as we cloned the whole dha operon with all the sequences between individual genes. However, in a heterologous expression system the expression of individual genes may differ from that in the natural host $(C$. butyricum). An analysis of DNA sequences upstream of the dha genes has revealed some departures from the consensus Shine-Dalgarno sequence of E. coli. Thus, the recognition of the ribosome binding sites of the C. butyricum dha genes by $E$. coli translation machinery could be less effective (Makrides, 1996; Mironova et al., 1999; Shultzaberger et al., 2001). However, above the difficulty could probably be overcome by cloning a gene encoding a DhaT isoenzyme more active than DhaT from C. butyricum e.g. YqhD from E. coli, encoding a NADPH-dependent 1,3PDO dehydrogenase (Emptage et al., 2009). YqhD utilizes NADPH rather than NADH and it is likely that the differences in the cofactor reduced/oxidized ratios contribute to the higher production of 1,3-PDO by this enzyme (Nakamura \& Whited, 2003).

Media containing glycerol or glycerol-glucose mixture as the carbon source were tested. E. coli cells used glucose preferentially (not shown), which resulted in no or lower 1,3-PDO production (Fig. 3). Moreover, the dha operon is repressed by glucose (Sprenger et al., 1989). Therfore, when glycerol was used as sole carbon source, the obtained 1,3-PDO amount was 1.1 and 13.2-times higher than in glycerol-glucose medium for $E$. coli BL21(DE3) and E. coli Rosetta2(DE3), respectively (Fig. 3).

Fermentation by recombinant E. coli strains was performed in anaerobic flasks, without shaking, but the media used were not reduced before. Thus, it is possible that, in such conditions a part of glycerol is dehydrogenated by the glycerol dehydrogenase (DhaD) of E. coli (Sprenger et al., 1989). Consequently, low 1,3-PDO concentration and yield was observed $-3.7 \mathrm{~g} \cdot \mathrm{l}^{-1}$ and $0.3 \mathrm{~g}$ per $1 \mathrm{~g}$ of glycerol consumed, respectively, for induced expression in E. coli Rosetta2(DE3) (Fig. 3, 4). On the other hand, culturing of the recombinant E. coli in strict anaerobic conditions failed to produce satisfying results because the strain was not able to grow (not shown).

One way to improve 1,3-PDO production in E. coli could be construction of a double-induced expression system, with a delayed dhaB1 and B2 induction. DhaT may be also cloned under control of a stronger promotor than that of the dhaB1B2 genes.

Is was observed that DhaT from C. freundii expressed in E. coli was more active in the presence of $50 \mu \mathrm{M}$ $\mathrm{Fe}^{2+}$ (Daniel et al., 1995). However, there is no such information for its isoenzymes from Clostridium sp. On the other hand, iron limitation causes higher 1,3-PDO production by Clostridium sp., probably by inhibiton of other dehydrogenases involved in the formation of butanol and ethanol (Dabrock et al., 1992; Raynaud et al., 2003; O'Brien et al., 2004). We therefore checked if supplementing the medium with $\mathrm{Fe}^{2+}$ up to $100 \mu \mathrm{M}$ could improve 1,3-PDO production, but no such effect was obtained. One of the reasons for this lack of improvement could be the absence of competing dehydrogenases in the heterologous system used.

Several papers describing construction of 1,3-PDOproducing E. coli strains have been published. Emptage et al. (2009) constructed an E. coli strain producing up to $130 \mathrm{~g} \cdot 1^{-1} 1,3$-PDO. However, in there expression system glucose was used as the sole carbon and energy source. Moreover, the recombinant E. coli strain harboured genes of the dha operon of $K$. pneumoniae and so $\mathrm{B}_{12}$ vitamin had to be added to the medium to activate the glycerol dehydratase. Glycerol was used as the sole carbon source was obtained by others (Skraly et al., 1998) and $6.3 \mathrm{~g} \cdot \mathrm{l}^{-1}$ of 1,3-PDO was produced, again for $E$. coli expressing the $K$. pneumoniae dha genes.

For comparision, a single-stage culture of $C$. butyricum with raw glycerol produced up to 35-48 g of 1,3-PDO per litre with a yield of $0.55 \mathrm{~g}$ per one gram of glycerol (Papanikolaou et al., 2000; Chatzifragkou et al., 2011). Glycerol fermentation by the C. butyricum 2CR371.5, which was the source of the genes studied here, results in $0.57 \mathrm{~g}$ of $1,3-\mathrm{PDO}$ per $1 \mathrm{~g}$ of glycerol consumed (Dabrowski et al., 2012).

The recombinant E. coli strains expressing genes of the dha operon from C. butyricum 2CR371.5 did not give satisfying results due to the low 1,3-PDO production level and long time required for maximal production. We believe it should be possible to obtain much more efficient glycerol fermentation to 1,3-PDO by E. coli expressing genes of the 1,3-propanediol operon from C. butyricum 2CR371.5 if modifications discussed above are introduced. That effort should be worthwhile, because 1,3-PDO is a desired chemical and the costs of its synthesis are still high.

\section{Acknowledgements}

This work was financially supported by the $7^{\text {th }}$ Framework of the European Union in the research area ENERGY-2007-3.3-02 — New uses for glycerine in biorefineries, project GLYFINERY (no. 213506). 


\section{REFERENCES}

Barbirato F, Grivet JP, Soucaille P, Bories A (1996) 3-hydroxypropionaldehyde, an inhibitory metabolite of glycerol fermentation to 1,3-propanediol by enterobacterial species. Appl Envir Microbiol 62: 1448-1451.

Barbirato F, Soucaille P, Bories A (1996) Physiologic mechanisms involved in accumulation of 3-hydroxypropionaldehyde during fermentation of glycerol by Enterobacter agglomerans. Appl Envir Microbiol 62: 4405-4409.

Boenigk R, Bowien S, Gottschalk G (1993) Fermentation of glycerol to 1,3-propanediol in continuous cultures of Citrobacter freundii. Appl Microbiol Biotechnol 38: 453-457.

Burch RR, Dorsch RR, Laffend LA, Nagarajan V, Nakamura C (2007) Bioconversion of a fermentable carbon source to 1,3-propanediol by a single microorganism. US patent, 7,169,588 B2

Burkhard O, Grunwaldt E, Mahmoud O, Jennewein S (2009) Genome shuffling in Clostridium diolis DSM 15410 for improved 1,3-propanediol production. Appl Envir Microbiol 75: 7610-7616.

Chatzifragkou A, Papanikolaou S, Dietz D, Doulgeraki AI, Nychas GJ, Zeng AF (2011) Production of 1,3-propanediol by Clostridium butyricum growing on biodiesel-derived crude glycerol through a nonsterilized fermentation process. Appl Microbiol Biotechnol 91: 101-112.

Dabrock G, Bahl H, Gottschalk G (1992) Parameters affecting solvent production by Clostridium pasteurianum. Appl Envir Microbiol 58: 1233-1239.

Daniel R, Boenigk R, Gottschalk G (1995) Purification of 1,3-propanediol dehydrogenase from Citrobacter freundii and cloning, sequencing, and overexpression of the corresponding gene in Escherichia coli.j Bacteriol 177: 2151-2156.

Dąbrowski S, Zabłotna E, Pietrewicz-Kubicz D, Długołęcka A (2012) Screening of environmental samples for bacteria producing 1,3-propanediol from glycerol. Acta Biochim Pol 59: 000-000.

Eliot AC, Gatenby AA, Van Dyk TK (2011) Recombinant bacteria for producing glycerol and glycerol-derived products from sucrose. Patent application, US 2011/0136190.

Emptage M, Haynie SL, Laffend LA, Pucci JP, Whited G (2009) Process for the biological production of 1,3-propanediol with high titter. Patent application, US 2009/0253192.

Gungormusler M, Gonen C, Ozdemir G, Azbar N (2010) 1,3-Propanediol production potential of Clostridium saccharobutylicum NRRL B-643. $N$ Biotechnol 27: 782-788.

Gupta A, Murarka A, Campbell P, Gonzales R (2009) Anaerobic fermentation of glycerol in Paenibacillus macerans: methabolic pathway and environmental determinants. Appl Envir Microbiol 75: 5871-5883.

Hao J, Lin R, Zheng Z, Sun Y, Liu D (2008) 3-hydroxypropionaldehyde guided glycerol feeding strategy in aerobic 1,3-propanediol production by Klebsiella pneumoniae. I Ind Microbiol Biotechnol 35: 16151624.

Hao J, Wang W, Tian J, Li J, Liu D (2008) Decrease of 3-hydroxypropionaldehyde accumulation in 1,3-propanediol production by overexpressing dhaT gene in Klebsiella pneumoniae TUAC01. I Ind Microbiol Biotechnol 35: 735-741.

Kane JF (1995) Effects of rare codon clusters on high-level expression of heterologous proteins in Escherichia coli. Curr Opin Biotechnol 6: 494-500.

Krauter A, Willke T, Vorlop K-D (2012) Production of high amounts of 3-hydroxypropionaldehyde from glycerol by Lactobacillus reuteri with strongly increased biocatalyst lifetime and productivity. $N$ Biotechnol 29: 211-217.

Liu H, Xu Y, Zheng Z, Liu D (2010) 1,3-Propanediol and its copolymers: research, development and industrialization. Biotechnol J 5: 1137-1148.

Luo LH, Seo JW, Baek JO, Oh BR, Heo SY, Hong WK, Kim $\mathrm{DH}, \mathrm{Kim} \mathrm{CH}$ (2011) Identification and characterization of the propanediol utilization protein PduP of Lactobacillus reuteri for 3-hydroxypropionic acid production from glycerol. Appl Microbiol Biotechnol 89: $697-703$.
Marçal D, Rêgo AT, Carrondo MA, Enguita FJ (2009) 1,3-propanediol dehydrogenase from Klebsiella pneumoniae: decameric quaternary structure and possible subunit cooperativity. J Bacteriol 191: 1143-1151.

Makrides SC (1996) Strategies for achieving high-level expression of genes in Escherichia coli. Microbiol Rev 60: 512-538.

Mironova RS, Xu J, AbouHaidar MG, Ivanov IG (1999) Efficiency of a novel non-Shine-Dalgarno and a Shine-Dalgarno consensus sequence to initiate translation in Escherichia coli of genes with different downstream box composition. Microbiol Res 54: 35-41.

Mu Y, Teng H, Zhang D-J, Wang W, Xiu Z-L (2006) Microbial production of 1,3-propanediol by Klebsiella pneumoniae using crude glycerol from biodiesel preparations. Biotechnol Lett 28: 1755-1759.

Murarka A, Dharmadi Y, Yazdani SS, Gonzalez R (2008) Fermentative utilization of glycerol by Escherichia coli and its implications for the productions of fuels and chemicals. Appl Environ Microbiol 74: 1124-1135.

Nagarajan V, Nakamura CE (1997) Production of 1,3-propanediol from glycerol by recombinant bacteria expressing recombinant diol dehydratase. US patent, 5,633,362.

Nakamura CE, Whited GM (2003) Metabolic engineering for the microbial production of 1,3-propanediol. Curr Opin Biotechnol 14: 454 459 .

Nevoigt E (2008) Progress in metabolic engineering of Saccharomyces cerevisiae. Microbiol Mol Biol Rev 72: 379-412.

Nishizawa A, Nakayama M, Uemura T, Fukuda Y, Kimura S (2010) Ribosome-binding site interference caused by Shine-Dalgarno-like nucleotide sequences in Escherichia coli cells. J Biochem 147: 433-443.

O’Brien JR, Raynaud C, Croux C, Girbal L, Soucaille P, Lanzilotta WN (2004) Insight into the mechanism of the B12-independent glycerol dehydratase from Clostridium butyricum: preliminary biochemical and structural characterization. Biochemistry 43: 4635-4645.

Papanikolaou S, Ruiz-Sanchez P, Pariset B, Blanchard F, Fick M (2000) High production of 1,3-propanediol from industrial glycerol by a newly isolated Clostridium butyricum strain. J Biotechnol 77: 191-208.

Pasteris SE, Strasser de Saad AM (2009) Sugar-glycerol cofermentations by Lactobacillus hilgardii isolated from wine. I Agric Food Chem 57: 3853-3858.

Raynaud C, Sarçabal P, Meynial-Salles I,Croux C, Soucaille P (2003) Molecular characterization of the 1,3-propanediol (1,3-PD) operon of Clostridium butyricum. Proc Natl Acad Sci US A 100: 5010-5015.

Saint-Amans S, Girbal L, Andrade J, Ahrens K, Soucaille P (2001) Regulation of carbon and electron flow in Clostridium butyricum VPI 3266 grown on glucose-glycerol mixtures. I Bacteriol 183: 1748-1754.

Sambrook J, Russel DW (2001) Molecular cloning: a laboratory manual. Cold Spring Harbor Laboratory Press, New York.

Shultzaberger RK, Bucheimer RE, Rudd KE, Schneider TD (2001) Anatomy of Escherichia coli ribosome binding sites. J Mol Biol 313: 215-228

Skraly FA, Lytle BL, Cameron DC (1998) Construction and characterization of a 1,3-propanediol operon. Appl Environ Microbiol 64: 98-105.

Sprenger GA, Hammer BA, Johnson EA, Lin ECC (1989) Anaerobic growth of Escherichia coli on glycerol by importing genes of the dha operon from Klebsiella pneumoniae. Microbiology 135: 1255-1262.

Tong IT, Liao HH, Cameron DC (1991) 1,3-propanediol production by Escherichia coli expressing genes from the Klebsiella pneumoniae dha regulon. Appl Environ Microbiol 57: 3541-3546.

Yazdani SS, Gonzalez R (2007) Anaerobic fermentation of glycerol: a path to economic viability for the biofuels industry. Curr Opin Biotechnol 18: 213-219.

Zeng AP, Biebl H (2002) Bulk chemicals from biotechnology: the case of 1,3-propanediol production and the new trends. Adv Biochem Eng Biotechnol 74: 239-259.

Zheng ZM,Hu QL, Hao J,Xu F, Guo NN, Sun Y, Liu DH (2008) Statistical optimization of culture conditions for 1,3-propanediol by Klebsiella pneumoniae AC 15 via central composite design. Bioresour Technol 99: 1052-1056. 
